\title{
Papel da imunoistoquímica no diagnóstico das alterações oculares na leishmaniose tegumentar americana. Relato clínico-patológico de cinco casos
}

\section{Role of imunohistochemistry in the diagnosis of ocular lesions in mucocutaneous leishmaniasis. Report of five cases}

Lívia Maria Nossa Moitinho', Luiz Antônio Rodrigues de Freitas², Eduardo Ferrari Marback², Roberto Lorens Marback ${ }^{4}$

\section{Resumo}

Objetivo: Descrever os achados clínicos, histopatológicos e imunoistoquímicos de cinco pacientes portadores de leishmaniose tegumentar americana com manifestações oftalmológicas. Métodos: Estudo retrospectivo, no período de quatro anos, compreendendo análise dos prontuários de pacientes do Hospital Universitário Professor Edgard Santos e das lâminas de histopatologia e do estudo imunoistoquímico.Resultado: $\mathrm{O}$ sintoma mais frequente foi a hiperemia conjuntival (2/5), seguida por lesão crostosa palpebral (1/5), leucoplasia conjuntival (1/5) e prurido ocular (1/5). Todos os pacientes foram submetidos à biópsia. O estudo histológico evidenciou inflamação crônica (3/5) e hiperplasia pseudoepiteliomatosa (2/5). Todos os pacientes com aspecto histopatológico de hiperplasia pseudoepiteliomatosa apresentaram resultado positivo para leishmaniose pela imunoistoquímica (anticorpo policlonal antileishmania). Conclusão: Devido ao polimorfismo das manifestações oculares da leishmaniose tegumentar americana, à inespecificidade do quadro histopatológico e à grande dificuldade de visualização de amastigotas na coloração hematoxilina-eosina, a biópsia com estudo imunoistoquímico contribuiu para a confirmação diagnóstica.

Descritores: Leishmaniose/patologia; Infecções oculares parasitárias/diagnóstico; Doenças oculares/micorbiologia; Imunoistoquímica; Relatos de casos

\footnotetext{
${ }^{1}$ Mestre, Professora assistente da Fundação Bahiana para Desenvolvimento das Ciências - FBDC - Salvador (BA), Brasil; ${ }^{2}$ Doutor, Professor Adjunto da Universidade Federal da Bahia e Pesquisador da Fundação Oswaldo Cruz. Salvador (BA), Brasil; ${ }^{3}$ Doutor, Médico Oftalmologista do Hospital Universitário Professor Edgard Santos da Universidade Federal da Bahia - UFBA Salvador (BA), Brasil;

${ }^{4}$ Doutor, Professor Titular Oftalmologia. Hospital Universitário Professor Edgard Santos da Universidade Federal da Bahia - UFBA Salvador (BA), Brasil.
} 


\section{INTRODUÇÃO}

$\mathbf{L}$ eishmaniose envolve um espectro de doenças que ocorrem mais comumente em áreas tropicais e subtropicais, apresenta incidência de 500.000 e prevalência de 2.4 milhões de $\operatorname{casos}^{(1)}$. No Brasil, é endêmica, com aproximadamente 30.000 casos notificados por ano ${ }^{(2)}$.

Segundo Marback ${ }^{(3)}$, a ocorrência de lesões oculares na leishmaniose tegumentar americana foi descrita pela primeira vez por Pina em 1912. As mais comuns manifestações clínicas da doença são: blefarite, conjuntivite, ceratoconjuntivite e lesão erosiva palpebral $^{(3-8)}$. Outros tipos de manifestação oftalmológica são a simulação de dacriocistite, chalázio, tumores como ceratoacantoma e carcinoma basocelular, estenose do ducto lacrimo-nasal e uveíte ${ }^{(9-11)}$.

Aspectos epidemiológicos e o teste cutâneo positivo para leishmania são importantes para o diagnóstico. A confirmação diagnóstica, atualmente, pode ser obtida através da demonstração de antígenos de Leishmania no tecido por técnica imunoistoquímica. ${ }^{12}$ Esta técnica utiliza anticorpo policlonal antileishmania e marca positivamente antígenos de Leishmania, sobretudo em macrófagos e células endoteliais ${ }^{(13)}$.

O objetivo deste trabalho foi descrever a análise clínica e histopatológica de cinco casos de leishmaniose tegumentar americana com manifestações oftalmológicas, submetidos à biópsia, enfatizando o papel da imunoistoquímica no diagnóstico.

\section{Apresentações dos casos}

Foram estudados retrospectivamente, cinco casos de pacientes portadores de leishmaniose tegumentar americana, encaminhados para o Ambulatório de Oftalmologia do Hospital Universitário Professor Edgard Santos, no período de quatro anos.

Os aspectos clínicos como: idade, gênero, sinais e sintomas, tempo de doença e suspeita diagnóstica são apresentados na Tabela 1. Os achados histopatológicos e imunoistoquímicos, conduta e terapêutica podem ser apreciadao na Tabela 2.

\section{Resultados}

A idade dos pacientes variou de 8 a 72 anos (média 32,4$)$. Três pacientes foram do gênero feminino e 2 , do masculino.

O tempo de doença variou de 3 a 240 dias (média $81,6)$. O sintoma mais frequente foi a hiperemia conjuntival

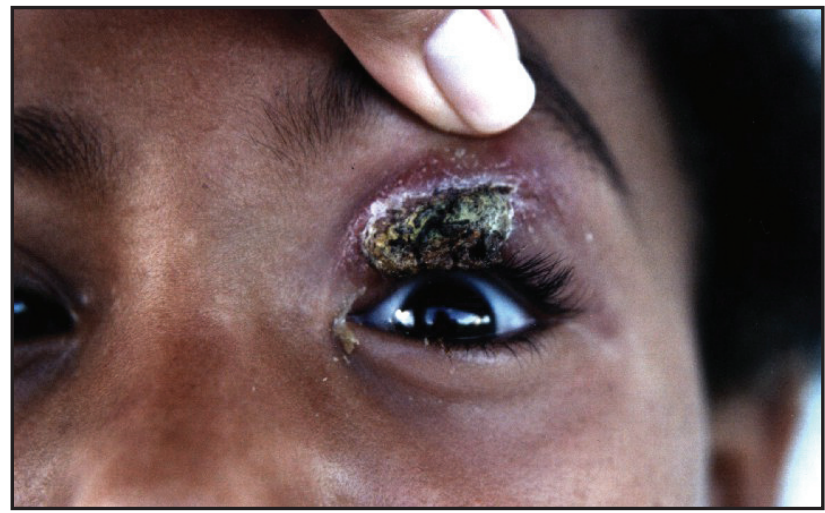

Figura 1: Lesão ulcerada, com formação de crosta envolvendo grande extensão da pálpebra superior esquerda

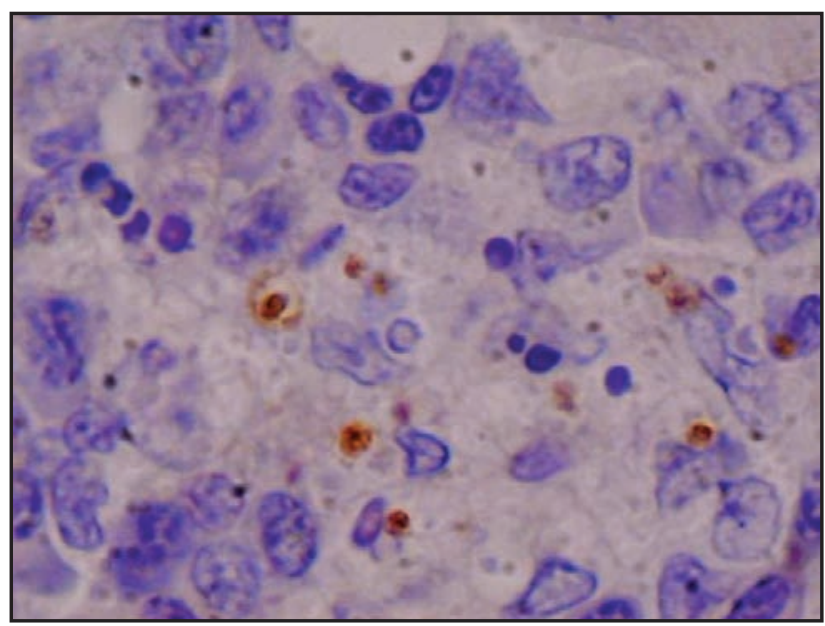

Figura 2: Microfotografia - A imunoistoquímica evidencia amastigotas marcadas pelo antígeno para leishmania

(40\%), seguida por lesão crostosa palpebral (20\%) (Figura 1), leucoplasia conjuntival (20\%) e prurido ocular (20\%). Nenhum caso teve a suspeita de leishmaniose nas manifestações oftalmológicas. Em um caso o aspecto clínico foi muito sugestivo de carcinoma epidermóide de conjuntiva.

Todos os pacientes foram submetidos à biópsia, inclusive os casos com suspeita de conjuntivite devido ao fato de os pacientes já apresentarem diagnóstico clínico de leishmaniose tegumentar americana, inclusive com positividade à reação de Montenegro.

$\mathrm{O}$ aspecto histopatológico em três pacientes (60\%) foi inflamação crônica inespecífica e em dois (40\%) hiperplasia pseudoepiteliomatosa (Tabela 2).

Os pacientes, que apresentaram hiperplasia pseudoepiteliomatosa, mostraram igualmente resultado positivo pela imunoistoquímica (Figura 2).

Apenas 1 paciente não respondeu ao antimonial pentavalente como terapêutica. Neste caso foi utilizada a Anfotericina B com melhora sintomatológica. 
Tabela 1

Idade, gênero, tempo de doença, sinais e sintomas e suspeita clínica dos pacientes estudados

\begin{tabular}{ccccll}
\hline Paciente & Idade & Gênero & Tempo de doença & Sinais/ Sintomas & Suspeita diagnóstica \\
\hline 1 & 32 & Feminino & 3 dias & Hiperemia conj. OE & Conjuntivite \\
2 & 12 & Feminino & $30 \mathrm{dias}$ & Lesão crostosa palp. OD & Blastomicose Sulamericana \\
3 & 38 & Masculino & 8 meses & Leucoplasia conj. OD & Carcinoma Epidermóide \\
4 & 72 & Masculino & 15 dias & Hiperemia conj. AO & Conjuntivite \\
5 & 8 & Feminino & 4 meses & Prurido OD & Conjuntivite \\
\hline
\end{tabular}

$\mathrm{OD}=$ olho direito; $\mathrm{OE}=$ olho esquerdo

Tabela 2

Conduta, achados histopatológicos e imunoistoquímicos e terapêutica dos pacientes estudados

\begin{tabular}{|c|c|c|c|c|}
\hline Paciente & Conduta & Histopatologia & Imunoistoquímica & Terapêutica \\
\hline 1 & $\begin{array}{l}\text { Biópsia } \\
\text { incisional }\end{array}$ & Inflamação crônica & Negativa & $\begin{array}{l}\text { Antimonial } \\
\text { pentavalente }\end{array}$ \\
\hline 2 & $\begin{array}{l}\text { Biópsia } \\
\text { incisional }\end{array}$ & Hiperplasia pseudoepiteliomatosa & Positiva $3+++$ & $\begin{array}{l}\text { Antimonial } \\
\text { pentavalente }\end{array}$ \\
\hline 3 & $\begin{array}{l}\text { Biópsia } \\
\text { excisional }\end{array}$ & Hiperplasia pseudoepiteliomatosa & Positiva $1+$ & $\begin{array}{l}\text { Antimonial } \\
\text { pentavalente }\end{array}$ \\
\hline 4 & $\begin{array}{l}\text { Biópsia } \\
\text { incisional }\end{array}$ & Inflamação crônica. & Positiva $1+$ & Anfotericina B* \\
\hline 5 & $\begin{array}{l}\text { Biópsia } \\
\text { incisional }\end{array}$ & Inflamação crônica & Negativa & $\begin{array}{l}\text { Antimonial } \\
\text { pentavalente }\end{array}$ \\
\hline
\end{tabular}

* A Anfotericina B foi utilizada como segundo tratamento, após falência do antimonial pentavalente

\section{DiscuSSÃO}

As manifestações oculares da leishmaniose tegumentar americana mais comumente descritas na literatura são: lesões palpebrais ${ }^{(3,8,10)}$, conjuntivite $^{(3,5)}$, blefarite $^{(4,6,7)}$ e uveíte ${ }^{(3)}$.

A maior série de casos de alterações oculares na leishmaniose tegumentar americana encontrada na literatura brasileira foi publicada em $1953^{3}$. Foram estudados 19 pacientes, destes 11 (57,9\%) eram do gênero feminino e $8(42,1 \%)$ do masculino. Nesse estudo, ele observou: $8(42,1 \%)$ casos de conjuntivite, $5(26,3 \%)$ casos de ceratite, $4(21 \%)$ feridas em pálpebra, $2(10,5 \%)$ dacriocistites e $1(5,3 \%)$ blefarite. Todos os pacientes obtiveram melhora clínica com o uso de terapia antimonial. Na nossa série, observamos $3(60 \%)$ pacientes do gênero feminino e 2 (40\%) do masculino. O sintoma mais freqüente também foi a hiperemia conjuntival $(40 \%)$ e todos, com exceção de apenas um caso, responderam bem à terapia com antimonial.

Recentemente, o estudo imunoistoquímico ${ }^{13}$ tem sido utilizado na confirmação diagnóstica da leishmaniose tegumentar americana, pois os achados à microscopia óptica frequentemente são inespecíficos, representados por inflamação crônica ou reação granulomatosa, sendo os parasitas raramente identificáveis $^{(12)}$. Positividade das biópsias em mucosa nasal, através da análise imunoistoquímica é encontrada em $62 \%$ a $75 \%$ dos $\operatorname{casos}^{(14)}$. Dado esse, compatível com o encontrado em nossa pequena série. Não foram encontrados, em nossa pesquisa, estudos sobre a utilização da imunoistoquímica para diagnóstico de leishmaniose em lesões oftalmológicas.

Devido ao fato de se tratar de uma doença endêmica no Brasil, com grande polimorfismo de manifestações oculares, o conhecimento dessas apresentações clínicas, bem como, o diagnóstico preciso e precoce são fundamentais para minimizar o risco de perda visual e cicatrizes viciosas nesses pacientes.

\section{Abstract}

Purpose: To describe the clinical, histopathological and immunoistochemical findings of ocular leishmaniasis in five patients presenting positive Montenegro reaction. Methods: Retrospective study in four years of the clinical 
charts, histopathologic and immunoistochemic study of the material obtained by biopsy of ocular lesions in five patients with positive Montenegro reaction. Results: The most frequent clinical findings was conjunctival hyperemia (2/ 5), followed by crusting lid lesion (1/5), conjunctival leukoplakia (1/5) and ocular itching (1/5). The histopathological study disclosed chronic inflammation (3/ 5) and pseudoepitheliomatous hyperplasia (2/5). The all patients that presented pseudoepitheliomatous hyperplasia in the biopsy material also presented immunohistochemical positivity for polyclonal antibody anti-leishmania. Conclusion: The immunoistochemical study was contribuitory for the diagnosis of ocular leishmaniasis in the five patients of this study.

Keywords: Leishmaniasis/pathology; Eye infections, parasitic/diagnosis; Eye diseases/microbiology; Immunohistochemistry; Case reports

\section{RefERÊNCIAS}

1. Desjeux P. Leishmaniasis: current situation and new perspectives. Comp Immunol Microbiol Infect Dis. 2004;27(5):305-18.

2. Brasil. Ministério da Saúde. Fundação Nacional de Saúde. Estudos e pesquisas em epidemiologia. Inf Epidemiol SUS. 1995;4(Supl 1):56.

3. Marback H. Lesões oculares da leishmaniose tegumentar americana [tese]. Salvador: Faculdade de Medicina da Universidade Federal da Bahia; 1953.

4. Longstaffe JA, Jefferies AR, Kelly DF, Bedford PG, Herrtage ME, Darke PG. Leishmaniasis in imported dogs in the United Kingdom; a potential health hazard. J Small Anim Pract. 1983;24(1):23-30.

5. Slappendel RJ. Canine leishmaniasis. A review based on 95 cases in The Netherlands. Vet Q. 1988;10(1):1-16.

6. Boldy KL, Clerc B. Ocular manifestation of systemic disease. In: Ettinger SJ, editor. Textbook of veterinary internal medicine: diseases of the dog and cat. 3rd ed. J. Philadelphia: W.B. Saunders; 1989. p. 75-84.
7. Bialasiewicz AA, Balmes R, Busse H. [Unilateral chronic granulomatous blepharitis as a leading symptom of Oriental cutaneous leishmaniasis in Germany. Giesma stain as rapid diagnosis and initial description of systemic therapy with gamma-interferon]. Klin Monatsbl Augenheilkd. 1992;200(3):219-23. German.

8. Oliveira-Neto MP, Martins VJ, Mattos MS, Pirmez C, Brahin LR, Benchimol E. South American cutaneous leishmaniasis of the eyelids: report of five cases in Rio de Janeiro State, Brazil. Ophthalmology. 2000;107(1):169-72.

9. Baddini-Caramelli C, Matayoshi S, Moura EM, Araf D, Santo R, Voegels R, Kara-José N. Chronic dacryocystitis in American mucocutaneous leishmaniasis. Ophthal Plast Reconstr Surg. 2001;17(1):48-52.

10. Ferry AP. Cutaneous leishmaniasis (oriental sore) of the eyelid. Am J Ophthalmol. 1977;84(3):349-54.

11. Ferrari TC, Guedes AC, Oréfice F, Genaro O, Pinheiro SR, Marra MA, et al. Isolation of Leishmania sp. from aqueous humor of a patient with cutaneous disseminated leishmaniasis and bilateral iridocyclitis (preliminary report). Rev Inst Med Trop Sao Paulo. 1990;32(4):296-8.

12. Amato VS, Duarte MI, Nicodemo AC, de Carvalho LV, Pagliari C, da Matta VL, et al. An evaluation of clinical, serologic, anatomopathologic and immunohistochemical findings for fifteen patients with mucosal leishmaniasis before and after treatment. Rev Inst Med Trop Sao Paulo. 1998;40(1):23-30.

13. Fróes AM, dos Santos CV, Penha-Filho ML, Teixeira MC, Correa Silva TM, Oliveira GG, et al. Sub-clinical infection as an effective protocol for obtaining anti-Leishmania chagasi amastigote antibodies of different animal species. Vet Immunol Immunopathol. 2004;99(3-4):135-41.

14. Amato VS. Utilização do isotionato de pentamidina para o tratamento da leishmaniose mucosa [dissertação]. São Paulo: Faculdade de Medicina da Universidade de São Paulo; 1997.

ENDEREÇO PARA CORRESPONDÊNCIA

Roberto Lorens Marback

Rua Eduardo José dos Santos, $n^{\circ}$ 147, sala 808

Garibaldi

CEP 41940-455 - Salvador - BA - Brasil 